\title{
La chirurgie chez le patient atteint de la maladie à virus Ebola
}

1 y a 30 ans, à l'époque où j'étais stagiaire en chirurgie, on nous demandait de pratiquer une biopsie-exérèse des ganglions lymphatiques aux fins de diagnostic chez tous les patients atteints du sida. Nos professeurs jugeaient cette intervention futile et risquée. Mais nous nous pensions invincibles. On s'arrangeait pour pratiquer l'intervention tout seul, en portant un masque double, une blouse double et une double paire de gants. La couche de vêtements internes était censée permettre le retrait en toute sécurité de la couche de vêtements externes. Le linge à envoyer à la buanderie et les déchets étaient déposés dans des sacs doubles.

Encore une fois, nous sommes confrontés à un virus transmissible par contact avec un liquide organique et dont on ne connaît pas entièrement les risques. Seule une vaste expérience pourrait montrer clairement les mesures à prendre pour combattre la maladie à virus Ebola (MVE) en Afrique de l'Ouest. Nous avons très peu d'expérience pour décider des soins à administrer aux patients atteints de la MVE dans les pays industrialisés comme le Canada. Au début, on croyait que certaines mesures servant à maintenir artificiellement les fonctions vitales, comme la ventilation mécanique et l'hémodialyse, permettraient au patient atteint de la maladie à un stade avancé de se rétablir. Nous en sommes moins certains aujourd'hui. Notre confiance dans les protocoles de protection classiques est ébranlée. Dans le présent article, je tenterai d'examiner le rôle du chirurgien dans la prise en charge du patient atteint de la MVE.

Les protocoles des premiers soins à administrer dans les cas soupçonnés ou confirmés de MVE ont été élaborés et mis en pratique par des hôpitaux canadiens avec l'aide des provinces et de l'Agence de santé publique du Canada ${ }^{1}$. Le $\mathrm{D}^{\mathrm{r}}$ Robert Fowler a décrit cette maladie comme étant une affection gastro-intestinale accompagnée de fièvre, dont l'effet mortel sur l'équilibre hydroélectrolytique peut s'atténuer grâce à des soins intensifs modernes ${ }^{2}$. Au moment où j'écris cet article, on pense que 4 patients atteints de la MVE à un stade avancé ont été placés sous ventilateur mécanique et sous hémodialyse. On a réussi à éradiquer le virus chez 2 d'entre eux. Cette réussite nous incite à poursuivre nos efforts pour soutenir les patients présentant une insuffisance rénale et une insuffisance respiratoire causées par la MVE. Les chirurgiens sont souvent consultés dans le cas de patients gravement atteints d'une maladie similaire. Ils sont alors devant un dilemme: envisager une chirurgie chez un patient atteint de la MVE. Les connaissances s'accumulent rapidement dans ce domaine. Les équipes de soins cliniques valident leurs décisions au cas par cas. L'American College of Surgeons a adapté les lignes directrices des Centers for Disease Control and Prevention des États-Unis pour les interventions chirurgicales pratiquées chez des patients que l'on soupçonne atteints de la $\mathrm{MVE}^{3}$. Les chirurgiens devraient consulter à nouveau ces lignes directrices fréquemment pour prendre connaissance des mises à jour. À l'heure actuelle, les lignes directrices ne fournissent aucun renseignement en ce qui concerne les patients qui devraient subir une intervention chirurgicale.

Lorsqu'une intervention ou une chirurgie effractive est envisagée chez un patient atteint de la MVE, l'équipe de soins doit faire une analyze utilité-risque, qui doit non seulement tenir compte du point de vue du patient mais aussi de l'ensemble du milieu. D'autres modalités thérapeutiques et le report de l'intervention à plus tard doivent constituer pour l'instant les choix préférables. Il faut favoriser rigoureusement les méthodes de réhydratation par voie orale généralement acceptées plutôt que la réhydratation parentérale, et l'administration par voie périphérique plutôt que l'administration par voie veineuse centrale. Il faut réduire le plus possible le nombre d'analyses de sang et se limiter à celles qui s'effectuent au point d'intervention. Le nombre d'examens par imagerie devrait aussi être considérablement moins élevé chez les patients atteints de la MVE que chez les patients non atteints. L'implantation de cathéters artériels sera très difficilement justifiable. Pour réduire les risques de contact direct avec le patient, il faut utilizer une méthode automatisée et non effractive de surveillance de la tension artérielle et de saturation en oxygène.

Le plus cruel dilemme pour le chirurgien, c'est de juger si une chirurgie est futile lorsqu'on ne possède presque aucune expérience directe d'une maladie donnée. Une défaillance organique assombrit le pronostic chez les patients vivant dans des régions où les ressources du système de santé sont limitées, comme dans les pays où des flambées de la maladie sont observées actuellement. Dans les pays industrialisés, le pronostic est sombre à la défaillance d'un organe, notamment si celle-ci survient après le rétablissement de l'équilibre hydroélectrolytique et acidobasique. Une insuffisance hépatique et la nécrose ont été observées chez des patients atteints de la MVE. L'échec des mesures de maintien des fonctions vitales fait perdre tout espoir de survie; ni une greffe de foie ni l'implantation d'un dispositif d'assistance hépatique ne devraient être envisagées. La chirurgie en cas de péritonite, d'hémorragie gastro-intestinale, de perforation intestinale ou d'ischémie 
intestinale risque de se solder par un échec. La maladie à virus Ebola risque d'entrainer une anasarque, accompagnée du syndrome du compartiment abdominal; si c'est le cas, une ventilation mécanique, un relâchement musculaire complet et une hémodialyse sont préférables à une laparotomie. La réanimation cardiopulmonaire n'est pas indiquée dans les cas de maladie au stade terminal. Malheureusement, l'expérience acquise en Afrique montre que les femmes enceintes atteintes de la MVE semblent exposées à un risque accru de fausse-couche et d'hémorragie associée à la grossesse. Les nouveau-nés de mères atteintes de la MVE n'ont pas survécu'.

L'endoscopie (bronchique ou gastro-intestinale) est une intervention très risquée chez les patients atteints de la MVE, car elle génère une forte expulsion d'aérosols à proximité de l'intervenant. L'endoscopie diagnostique échouera probablement à l'analyse utilité-risque, tout comme la plupart des interventions à objectif thérapeutique. Il faut tenir compte des risques accrus liés à la production d'aérosols si l'on a recours à la voie laparoscopique pour une chirurgie au thorax ou à l'abdomen. De même, il faut limiter l'utilisation des drains externes en raison des risques associés à la présence extracorporelle des liquides organiques.

La ventilation mécanique peut rendre une chirurgie nécessaire : la trachéotomie devrait être retardée jusqu'à ce que le patient ait éliminé le virus. L'oxygénation extracorporelle n'a probablement aucune chance de résister au test de l'analyze avantage-risques. La thoracotomie, tout comme l'intubation endotrachéale, devrait être considérée comme une intervention générant des aérosols. On devrait réduire le risque de contact avec les aérosols en portant un respirateur no 95 bien ajusté et en demandant à toutes les personnes dont la présence n'est pas essentielle de quitter le bloc opératoire. Après l'intervention chirurgicale, les aires avoisinantes devront être décontaminées.

Pour combattre la transmission du virus Ebola, on doit renforcer les protocoles de protection classiques. Certains éléments des protocoles militaires contre l'exposition à des substances chimiques, biologiques, radiologiques et nucléaires pourraient se révéler utiles. L'équipement de protection individuelle devrait comporter une blouse, une cagoule et des couvre-chaussures en combinaison d'une seule pièce. Les vérifications et l'aide par des compagnons de travail permettront de réduire les failles durant l'enfilage et le retrait de l'équipement de protection. La décontamination à l'aide de chiffons avant le retrait de l'équipement de protection réduit les risques de dissémination par inadvertance. En Afrique, d'héroïques équipes soignantes réutilizent les blouses et se douchent ensuite avec un agent de blanchiment dilué pour contenir la flambée de la maladie. Dans les pays industrialisés, il est essentiel de ne pas confondre les mesures de protection contre les bactéries causant la diarrhée, comme Clostridium difficile, avec les protocoles de protection contre la MVE. On peut devoir ajouter des blouses et des gants de chirurgien stériles aux combinaisons de protection contre la MVE qui, elles, ne sont pas stériles.

Pour éviter de répéter ce qui s'est fait dans les débuts du sida, les stagiaires en chirurgie et les jeunes chirurgiens ayant des enfants ne devraient pratiquer aucune intervention chez des patients atteints de la MVE. La tâche doit revenir aux chirurgiens d'expérience et l'équipe du bloc opératoire devrait être aussi réduite que possible. Le chirurgien appelé en consultation pour des patients atteints de la MVE ne devrait pas hésiter à demander conseil à un chirurgien d'un centre qui a de l'expérience. Après avoir surmonté la crainte suscitée par le sida, on a rapidement compris ce qu'était le VIH et comment le traiter. Comme bon nombre de chirurgiens, j'ai continué à pratiquer un vaste éventail de chirurgies, y compris des transplantations de foie, sur des patients infectés par le VIH. On a de bonnes raisons d'espérer que la crainte suscitée par la MVE finira par s'atténuer de la même façon. Ainsi, on sera en mesure d'administrer tout l'éventail des soins intensifs et chirurgicaux aux patients infectés par le virus Ebola.

\section{Vivian McAlister, MD \\ Co-rédacteur, fournal canadien de chirurgie}

Intérêts concurrents : Aucuns déclarés.

DOI: $10.1503 /$ cjs. 016014

\section{Références}

1. Canadian Critical Care Society, Canadian Association of Emergency Physicians, Association of Medical Microbiology \& Infectious Diseases Canada. Ebola clinical care guidelines: a guide for clinicians in Canada. Interim Report, August 29, 2014. Disponible : www.ammi .ca/media/69846/Ebola\%20Clinical\%20Care\%20Guidelines\%202\% 20Sep\%202014.pdf (accédé 2014 oct. 29).

2. Fowler RA, Fletcher T, Fischer WA II, et al. Caring for critically ill patients with Ebola virus disease. Perspectives from west Africa. Am 7 Respir Crit Care Med 2014;190:733-7.

3. Wren SM, Kushner AL. Surgical protocol for possible or confirmed Ebola cases. American College of Surgeons 7 October 2014. Disponible : www.facs.org/ebola/surgical-protocol (accédé 2014 oct. 29).

4. Jamieson DJ, Uyeki TM, Callaghan WM, et al. What obstetriciangynecologists should know about Ebola: a perspective from the Centers for Disease Control and Prevention. [Epub ahead of print]. Obstet Gynecol 2014. 